\title{
Orthostatic Hypotension and Risk of Cardiovascular Disease in Elderly People: The Rotterdam Study
}

\author{
Germaine C. Verwoert, ${ }^{* \dagger}$ Francesco U. S. Mattace-Raso, MD, PhD, ${ }^{* \dagger}$ Albert Hofman, MD, PhD, ${ }^{*}$ \\ Jan Heeringa, MD, ${ }^{*}$ Bruno H. C. Stricker, PhD, ${ }^{* \neq}$ Monique M. B. Breteler, MD, PhD, ${ }^{*}$ and \\ Jacqueline C. M. Witteman, PhD*
}

OBJECTIVES: To determine the prognostic role of orthostatic hypotension for cardiovascular disease (CVD) and all-cause mortality in elderly people.

DESIGN: Prospective study.

SETTING: Community based.

PARTICIPANTS: Five thousand sixty-four subjects from the Rotterdam study aged 55 and older.

MEASUREMENTS: Orthostatic hypotension was measured using a Dinamap automatic blood pressure recorder. Orthostatic hypotension is defined as a decline in systolic blood pressure of $20 \mathrm{mmHg}$ or more or a decline in diastolic blood pressure of $10 \mathrm{mmHg}$ or more from supine to standing position at any of three measurements taken 1,2 , and 3 minutes after standing.

RESULTS: At baseline, 901 subjects had orthostatic hypotension. During follow-up, 668 subjects had coronary heart disease (CHD) (mean follow-up $6.0 \pm 3.5$ years), and 1,835 subjects died (mean follow-up period $7.8 \pm 3.8$ years). Orthostatic hypotension increased the risk of CHD (hazard ratio $(\mathrm{HR})=1.31,95 \%$ confidence interval $(\mathrm{CI})=1.08$ 1.57 ) and all-cause mortality ( $\mathrm{HR}=1.22,95 \% \mathrm{CI}=1.09$ 1.36), in models adjusted for age and sex. The risk was slightly lower after additional adjustment for cardiovascular risk factors. In analyses stratified for age, the HRs for all-cause mortality were 1.80 (95\% CI 1.25-2.60), 1.13 $(0.89-1.42)$, and 1.27 (95\% CI $=1.11-1.44)$, in the first, second, and third tertile of age, respectively.

CONCLUSION: Orthostatic hypotension increases the risk of CHD and all-cause mortality in elderly people. The risk of CVD and mortality is strongest in younger and very old subjects. J Am Geriatr Soc 56:1816-1820, 2008.

From the * Department of Epidemiology and Biostatistics, ${ }^{\dagger}$ Section of Geriatric Medicine, Department of Internal Medicine; and ${ }^{\ddagger}$ Department of Internal Medicine, Erasmus University Medical Center, Rotterdam, the Netherlands.

Address correspondence to JCM Witteman, PhD, Department of Epidemio$\operatorname{logy} \&$ Biostatistics, Erasmus University Medical Center, PO Box 1738, 3000 DR Rotterdam, the Netherlands. E-mail: j.witteman@erasmusmc.nl

DOI: 10.1111/j.1532-5415.2008.01946.x
Key words: orthostatic hypotension; cardiovascular disease; all-cause mortality

$\mathrm{O}$ rthostatic hypotension is common in elderly people ${ }^{1-4}$ and is associated with syncope, ${ }^{5,6}$ falls, ${ }^{7}$ fractures, and potential morbidity, leading to functional impairment and hospitalization. ${ }^{8}$ Several studies have investigated the relationship between orthostatic hypotension and cardiovascular disease (CVD). Well-designed prospective, population-based, large-cohort studies have found that orthostatic hypotension increases the risk of stroke, ${ }^{9}$ coronary heart disease (CHD), ${ }^{10}$ and mortality ${ }^{11}$ in middle-aged subjects. Other studies have investigated the prognostic role of orthostatic hypotension in elderly people, although the results have been contradictory ${ }^{3,12-15}$ or performed in specific categories of patients. ${ }^{16-18}$ No previous study has investigated the relationship between orthostatic hypotension and the risk of heart failure.

The aim of the present study was to investigate whether orthostatic hypotension increases the risk of primary CVD and all-cause mortality in elderly people. The present study was performed within the framework of the Rotterdam Study, a large, ongoing, prospective, population-based study that included subjects aged 55 and older at baseline.

\section{METHODS}

\section{Study Population}

The Rotterdam Study is a prospective population-based cohort study comprising 7,983 subjects aged 55 and older living in Ommoord, a suburb of Rotterdam, the Netherlands. The overall aim of the Rotterdam Study is to assess the occurrence of and risk factors for chronic diseases in elderly people. The study design and objectives of the Rotterdam Study are described elsewhere. ${ }^{19}$ At baseline, a trained interviewer visited the 7,983 subjects at home to complete a computerized questionnaire. Participants visited 
the research center for the baseline examination from March 1990 to July 1993. During these visits at the research center, blood pressure measurements were obtained, and established cardiovascular risk factors were measured. The Medical Ethics Committee of Erasmus Medical Center approved the study, and written consent was obtained from all participants.

\section{Previous CVD and Cardiovascular Risk Factors}

A history of CHD, stroke, and heart failure was obtained through direct questioning and was considered positive when confirmed according to hospital discharge date or written information from the subject's general practitioner, as described previously. ${ }^{20-22} \mathrm{CHD}$ was defined as fatal or nonfatal myocardial infarction (MI), a percutaneous transluminal coronary angioplasty, a coronary artery bypass graft, sudden cardiac death, and death due to ventricular fibrillation and congestive heart failure. A history of MI was considered present in case of self-report of MI confirmed by electrocardiogram or additional clinical information or the presence of an electrocardiogram characteristic of prior MI. Information on smoking habits and the use of antihypertensive medication was obtained during the interview. Smoking was divided into three categories: current, former, and nonsmokers. Height and weight were measured, and body mass index $(\mathrm{BMI})$ was computed $\left(\mathrm{kg} / \mathrm{m}^{2}\right)$. Diabetes mellitus was defined as the use of blood glucose-lowering medication or a random or postload serum glucose level of $11.1 \mathrm{mmol} / \mathrm{L}$ or higher. ${ }^{23}$ Serum total cholesterol and highdensity lipoprotein cholesterol (HDL-C) values were determined using an automated enzymatic procedure (Boehringer Mannheim System, Mannheim, Germany). Two seated blood pressure measurements are obtained at the right brachial artery using a random zero sphygmomanometer. The mean of two consecutive measurements was used. Hypertension was defined as systolic blood pressure of $140 \mathrm{mmHg}$ or greater, diastolic blood pressure of $90 \mathrm{mmHg}$ or higher, or the use of antihypertensive medication. Orthostatic hypotension was measured using an automatic blood pressure recorder (Dinamap R, Tampa, FL). The baseline reading was the mean of two blood pressure measurements on the right upper arm with the subject in supine position after 5 minutes of rest. Measurements were repeated in the standing position after 1,2, and 3 minutes. Orthostatic hypotension was defined as a decline in systolic blood pressure of $20 \mathrm{mmHg}$ or more or a decline in diastolic blood pressure of $10 \mathrm{mmHg}$ or more from supine to standing position at any of the three measurements.

\section{Incident Cardiovascular Events and All-Cause Mortality}

Information on fatal and nonfatal cardiovascular outcomes was obtained through automated linkage with the files from general practitioners and letters and discharge reports from medical specialists. When a cardiovascular event was reported, the research assistants collected additional information from medical records of the general practitioner and, in addition, obtained information from the hospital discharge records or nursing home records, including letters from medical specialists. For the diagnosis of cardiac events, two research physicians independently coded all reported events. In the case of disagreement, a medical expert $(\mathrm{JH})$ in the field made a decision. In the case of stroke, two research physicians and an experienced neurologist coded the events. Codes were assigned according to the International Classification of Diseases, Tenth Revision (ICD-10). ${ }^{24} \mathrm{CHD}$ was defined as the occurrence of a fatal or nonfatal MI (ICD-10 code I21), a percutaneous transluminal coronary angioplasty, a coronary artery bypass graft, other form of acute (I24) or chronic ischemic heart disease (I25), sudden cardiac death (I46 and R96), or death due to ventricular fibrillation (I49) or congestive heart failure (I50). ICD-10 codes used for coding stroke were I61, I63, and I64. Heart failure was determined using a validated score that is similar to the definition of heart failure of the European Society of Cardiology. ${ }^{25}$ Information on vital status was acquired at regular intervals from the municipal authorities of Rotterdam. For the present study, follow-up was completed for stroke and CHD until January 2005 and for heart failure and all-cause mortality until October 2006.

\section{Population for Analysis}

Seven thousand nine hundred eighty-three subjects (response rate $78 \%$ ) agreed to participate and were interviewed at home, and 7,129 subjects visited the research center for physical examinations. Blood pressure measurements in the supine and standing positions were available in 6,455 participants. Subjects with CHD $(n=743)$, stroke $(\mathrm{n}=164)$, or heart failure $(\mathrm{n}=194)$ at baseline and subjects with missing data on smoking $(\mathrm{n}=357)$, use of antihypertensive medication $(\mathrm{n}=2,191)$, BMI $(\mathrm{n}=1,073)$, blood pressure $(\mathrm{n}=975)$, diabetes mellitus $(\mathrm{n}=202)$, total cholesterol $(\mathrm{n}=944)$, and HDL-C $(\mathrm{n}=973)$ were excluded from the analyses. The number with missing data is overlapping, leaving 5,064 subjects available for this analysis.

\section{Data Analysis}

The baseline characteristics of the subjects with and without orthostatic hypotension were compared using Student $t$-tests for continuous variables and chi-square tests for categorical variables. A power calculation was performed, which showed a power of $96 \%$ to reach a hazard ratio (HR) of 2 in a population of 5,064 subjects. Cox-proportional hazard models were used to estimate HRs with corresponding $95 \%$ confidence intervals (CIs) for CHD, stroke, heart failure, and all-cause mortality according to orthostatic hypotension status. The first model was adjusted for age and sex. Model 2 was additionally adjusted for smoking, use of antihypertensive medication, BMI, systolic blood pressure, diastolic blood pressure, diabetes mellitus, total cholesterol, and HDL-C. To investigate whether the prognostic role of orthostatic hypotension varied with aging together with the age-related changes of cardiovascular risk load, analyses were performed in tertiles of age, sex, hypertension status, and diabetes mellitus status. $P<.05$ was considered to be statistically significant. All analyses were performed using SPSS 11.0 statistical package for Windows 2000 (SPSS Inc., Chicago, IL).

\section{RESULTS}

The baseline characteristics of the 5,064 subjects are shown in Table 1. Thirty-eight percent of the subjects were men, 
Table 1. Baseline Characteristics of the Study Subjects $(\mathrm{N}=5,064)$

\begin{tabular}{|c|c|c|c|c|}
\hline Characteristic & $\begin{array}{c}\text { Total } \\
(\mathrm{N}=\mathbf{5 , 0 6 4 )}\end{array}$ & $\begin{array}{c}\text { Subjects without } \\
\mathrm{OH}(\mathrm{n}=4,163)\end{array}$ & $\begin{array}{l}\text { Subjects with } \mathrm{OH} \\
\quad(n=901)\end{array}$ & P-Value* \\
\hline Male, \% & 38.4 & 40.1 & 30.9 & $<.001$ \\
\hline \multicolumn{5}{|l|}{ Smoking, \% } \\
\hline Current & 22.4 & 22.3 & 23 & .67 \\
\hline Former & 41.6 & 42.6 & 37 & .002 \\
\hline Antihypertensive medication, \% & 26.9 & 25.3 & 34.3 & $<.001$ \\
\hline Body mass index, $\mathrm{kg} / \mathrm{m}^{2}$, mean $\pm \mathrm{SD}$ & $26.2 \pm 3.6$ & $26.2 \pm 3.5$ & $26.5 \pm 4.0$ & .04 \\
\hline Systolic blood pressure, $\mathrm{mmHg}$, mean $\pm \mathrm{SD}$ & $139 \pm 22$ & $138 \pm 22$ & $144 \pm 23$ & $<.001$ \\
\hline Diastolic blood pressure, $\mathrm{mmHg}$, mean $\pm \mathrm{SD}$ & $74 \pm 11$ & $74 \pm 11$ & $74 \pm 12$ & .94 \\
\hline Diabetes mellitus, \% & 8.9 & 8.3 & 11.5 & .002 \\
\hline
\end{tabular}

* Test of difference between means was performed using the Student $t$-test, and test of differences between proportions was performed using the chi-square test. $\mathrm{SD}=$ standard deviation; $\mathrm{OH}=$ orthostatic hypotension.

and the mean age was 68 . Of the 5,064 subjects in this study, $901(17.8 \%)$ had orthostatic hypotension; the prevalence of orthostatic hypotension was higher in women than in men $(P<.001)$. Subjects with orthostatic hypotension were older $(P<.001)$ and more likely to use antihypertensive medication $(P<.001)$ and had a higher BMI $(P=.04)$, higher systolic blood pressure $(P<.001)$, and higher prevalence of diabetes mellitus $(P=.002)$. During follow-up 668 had subjects CHD (mean follow-up period $6.0 \pm 3.5$ years), 503 subjects had a stroke (mean follow-up period $6.7 \pm 3.6$ years), 571 subjects developed heart failure (mean follow-up period $6.6 \pm 3.9$ years), and 1,835 subjects died (mean follow-up period $7.8 \pm 3.8$ years). Of the 901 subjects with orthostatic hypotension at baseline, 152 had CHD, 119 had stroke, 140 had heart failure, and 465 died during follow-up. In models adjusted for age and sex, orthostatic hypotension predicted CHD (HR $=1.31$, $95 \% \mathrm{CI}=1.08-1.57)$, heart failure $(\mathrm{HR}=1.21,95 \% \mathrm{CI}=$ $1.00-1.48)$, and all-cause mortality $(\mathrm{HR}=1.22,95 \%$ $\mathrm{CI}=1.09-1.36)$ (Table 2). The association between orthostatic hypotension and heart failure was less consistent in models adjusted for cardiovascular risk factors. Stratified analyses were performed to determine whether the association between orthostatic hypotension and incident events varied according to age, sex, hypertension status, and diabetes mellitus status. Mean age was 59.3 (range 55-63) in the first tertile, 67.0 (range 63.1-71.3) in the second tertile, and 78.0 (range 71.4-98.7) in the third tertile. Hypertension was present in $57.6 \%$ and diabetes mellitus in $8.9 \%$ of the participants. In the younger subjects (first tertile), orthostatic hypotension predicted all-cause mortality $(\mathrm{HR}=1.80,95 \% \mathrm{CI}=1.25-2.60)$ and showed a trend for $\mathrm{CHD}(\mathrm{HR}=1.56,95 \% \mathrm{CI}=0.99-2.46)$. In the older subjects (third tertile), orthostatic hypotension predicted stroke $(\mathrm{HR}=1.35,95 \% \mathrm{CI}=1.04-1.75)$, heart failure $(\mathrm{HR}=1.32,95 \% \mathrm{CI}=1.04-1.67)$, and all-cause mortality $(\mathrm{HR}=1.27,95 \% \mathrm{CI}=1.11-1.44)$ (Table 3$).$ In hypertensive participants, orthostatic hypotension increased the risk of $\mathrm{CHD}(\mathrm{HR}=1.27,95 \% \mathrm{CI}=1.03-1.57)$ and all-cause mortality $(\mathrm{HR}=1.20,95 \% \mathrm{CI}=1.06-1.36)$. In subjects with diabetes mellitus, orthostatic hypotension doubled the risk of heart failure ( $\mathrm{HR}=1.97,95 \% \mathrm{CI}=1.22-3.19)$.

\section{DISCUSSION}

In the present study, orthostatic hypotension slightly increased the risk of CHD and all-cause mortality in apparently healthy elderly people. Associations between orthostatic hypotension and cardiovascular risk were strongest in the younger and very old participants.

Previous studies have investigated the relationship between orthostatic hypotension and the risk of CHD, stroke,

Table 2. Cox Proportional Hazards Regression Models of Orthostatic Hypotension and Risk of Coronary Heart Disease (CHD), Stroke, Heart Failure, and All-Cause Mortality

\begin{tabular}{lcccc}
\hline & CHD (668/5,064) & Stroke (503/5,064) & Heart Failure (571/5,064) & All-Cause Mortality (1,835/5,064) \\
\cline { 2 - 5 } Model & \multicolumn{5}{c}{ Hazard Ratio $\mathbf{( 9 5 \%}$ Confidence Interval) $\boldsymbol{P}$-Value } \\
\hline 1 & $1.31(1.08-1.57) .005$ & $1.17(0.95-1.45) .13$ & $1.21(1.00-1.48) .05$ & $1.22(1.09-1.36)<.001$ \\
2 & $1.20(1.00-1.45) .05$ & $1.10(0.89-1.36) .35$ & $1.12(0.92-1.36) .27$ & $1.16(1.04-1.29) .007$ \\
\hline
\end{tabular}

Model 1 adjusted for age and sex.

Model 2 adjusted for age, sex, smoking, use of antihypertensive medication, body mass index, systolic blood pressure, diastolic blood pressure, diabetes mellitus, total cholesterol, and high-density lipoprotein cholesterol. 
Table 3. Stratified Proportional Hazard Models of Orthostatic Hypotension and Risk of Coronary Heart Disease, Stroke, Heart Failure, and All-Cause Mortality

\begin{tabular}{|c|c|c|c|c|}
\hline \multirow[b]{2}{*}{ Variable } & CHD & Stroke & Heart Failure & All-Cause Mortality \\
\hline & \multicolumn{4}{|c|}{ Events/Subjects; Hazard Ratio (95\% Confidence Interval) } \\
\hline Male & $343 / 1,946 ; 1.18(0.88-1.57)$ & $198 / 1,946 ; 1.09(0.76-1.58)$ & $249 / 1,946 ; 0.95(0.67-1.33)$ & $802 / 1,946 ; 1.27$ (1.07-1.51) \\
\hline Female & $325 / 3,118 ; 1.22(0.95-1.56)$ & $305 / 3,118 ; 1.10(0.85-1.42)$ & $322 / 3,118 ; 1.22(0.96-1.56)$ & $1,033 / 3,118 ; 1.10(0.95-1.26)$ \\
\hline \multicolumn{5}{|l|}{ Age tertile } \\
\hline 2 & $230 / 1,689 ; 1.14(0.81-1.62)$ & $169 / 1,689 ; 0.76(0.49-1.19)$ & $183 / 1,689 ; 0.91(0.61-1.37)$ & $501 / 1,689 ; 1.13(0.89-1.42)$ \\
\hline 3 & $284 / 1,687 ; 1.21(0.94-1.57)$ & $273 / 1,687 ; 1.35(1.04-1.75)$ & $324 / 1,687 ; 1.32(1.04-1.67)$ & $1,123 / 1,687 ; 1.27(1.11-1.44)$ \\
\hline \multicolumn{5}{|l|}{ Hypertension } \\
\hline No & 198/2,146; $0.93(0.61-1.40)$ & $129 / 2,146 ; 0.90(0.55-1.46)$ & $126 / 2,146 ; 0.99(0.61-1.60)$ & $564 / 2,146 ; 1.05(0.84-1.30)$ \\
\hline Yes & $470 / 2,918 ; 1.27$ (1.03-1.57) & $374 / 2,918 ; 1.14(0.90-1.44)$ & $445 / 2,918 ; 1.14(0.92-1.42)$ & $1,271 / 2,918 ; 1.20$ (1.06-1.36) \\
\hline
\end{tabular}

Note: Adjusted for age, sex, smoking, use of antihypertensive medication, body mass index, systolic blood pressure, diastolic blood pressure, diabetes mellitus, total cholesterol, and high-density lipoprotein cholesterol (when appropriate).

and mortality. The Atherosclerosis Risk in Communities Study, a large population-based prospective cohort study of middle-aged participants (aged 45-65), found strong associations between orthostatic hypotension and stroke, ${ }^{9}$ $\mathrm{CHD},{ }^{10}$ and mortality. ${ }^{11}$ Other studies have been performed in the older subjects. The Honolulu Heart Program, ${ }^{17}$ a prospective study of 3,741 Japanese men aged 71 to 93 , found that orthostatic hypotension increased the risk of all-cause mortality in elderly Japanese men. Other authors have shown that orthostatic hypotension increased the risk of MI and cardiovascular death in 700 homedwelling Finnish people aged 70 and older. ${ }^{12,14}$ On the contrary, in studies of 500 acute geriatric ward patients ${ }^{15}$ and 350 subjects aged 65 and older, ${ }^{3}$ orthostatic hypotension did not increase the risk of vascular death.

As previously reported, also in this large populationbased study, orthostatic hypotension increased the risk of CVD and all-cause mortality in elderly people. According to previous studies, ${ }^{10,18,26}$ subjects with orthostatic hypotension were older, used antihypertensive medication more often, had a higher mean BMI and higher mean levels of systolic blood pressure, and more often had diabetes mellitus.

The association between orthostatic hypotension and CVD may have several explanations. Orthostatic challenge displaces blood to the lower body. This could decrease thoracic blood volume $25 \%$ to $30 \% .^{27}$ The secondary reduction in coronary and cerebral flow during a strong postural blood pressure drop may cause myocardial and cerebral ischemia. ${ }^{3}$ Orthostatic hypotension could also be the expression of underlying CVD. Cerebral infarcts, MIs, and heart failure 28,29 could cause orthostatic hypotension. ${ }^{3}$ Also, diabetes mellitus ${ }^{30}$ and hypertension ${ }^{10}$ may be associated with orthostatic hypotension. Therefore, one possible question could be whether these conditions might explain the association between orthostatic hypotension and CVD. For this reason, subjects with previous CVD were excluded at baseline, and analyses adjusted for diabetes mellitus, hypertension, and other cardiovascular risk factors were performed. Nevertheless, associations between orthostatic hypotension and CHD and all-cause mortality remained statistically significant after adjustments, suggesting an independent prognostic role of orthostatic hypotension. The predictive role of orthostatic hypotension was strongest in younger and very old subjects. Some studies have suggested that orthostatic hypotension is a marker of frailty in the very old, ${ }^{17}$ whereas the prevalence of orthostatic hypotension is much lower in younger subjects. Therefore, it might be speculated that the presence of orthostatic hypotension in younger adults might be the expression of an underlying silent CVD.

It is known that hypertension is associated with orthostatic hypotension, ${ }^{4,26}$ particularly in elderly people, and that both might be the expression of blood pressure dysfunction; the present study found that, in hypertensive subjects, orthostatic hypotension increased the risk of CHD and all-cause mortality.

The present study has some limitations. First, blood pressure measurements were performed once, which could bias the results because of the daily variability of blood pressure. The use of multiple blood pressure measurements might have improved accuracy and precision. Second, measures of orthostatic hypotension and blood pressure were not available for all participants, although this will not have biased the results, because this was due almost entirely to logistic reasons and was therefore random. Third, the study subjects were Caucasian, so generalizability of the results might be difficult. Fourth, there could be some residual confounding. Orthostatic hypotension increases the risk of falls and consequent hip fractures, and these are associated with a high mortality rate, but because these variables were not adjusted for, this relationship cannot be assessed. Finally, no correction for multiple comparisons was performed, although it is unlikely that this biased the results. 
In conclusion, orthostatic hypotension increases the risk of CHD and all-cause mortality in apparently healthy elderly subjects. These findings support the call ${ }^{11}$ to understand the mechanisms responsible for cardiovascular morbidity and mortality in subjects with orthostatic hypotension. There is much evidence that orthostatic hypotension increases cardiovascular risk in elderly people. ${ }^{9-12,14,17}$ Future studies are needed to investigate whether identification and treatment of orthostatic hypotension can improve cardiovascular prognosis.

\section{ACKNOWLEDGMENTS}

\section{Conflict of Interest: None.}

Author Contributions: All authors contributed to the interpretation of the results and drafting of the manuscript. $\mathrm{AH}, \mathrm{MMB}, \mathrm{BHCS}$, and JCMW did conception and design of the study. GCV, FUSMR, and JCMW conducted statistical analysis and manuscript preparation.

\section{Sponsor's Role: None.}

\section{REFERENCES}

1. Caird FI, Andrews GR, Kennedy RD. Effect of posture on blood pressure in the elderly. Br Heart J 1973;35:527-530.

2. Mader SL, Josephson KR, Rubenstein LZ. Low prevalence of postural hypotension among community-dwelling elderly. JAMA 1987;258:1511-1514.

3. Raiha I, Luutonen S, Piha J et al. Prevalence, predisposing factors, and prognostic importance of postural hypotension. Arch Intern Med 1995;155:930935 .

4. Mattace-Raso FU, van der Cammen TJ, Knetsch AM et al. Arterial stiffness as the candidate underlying mechanism for postural blood pressure changes and orthostatic hypotension in older adults: The Rotterdam Study. J Hypertens 2006;24:339-344.

5. Lipsitz LA. Syncope in the elderly patient. Hosp Pract (Off Ed) 1986;21: 33-44.

6. Lipsitz LA, Pluchino FC, Wei JY et al. Syncope in institutionalized elderly: The impact of multiple pathological conditions and situational stress. J Chronic Dis 1986;39:619-630.

7. Tinetti ME, Williams TF, Mayewski R. Fall risk index for elderly patients based on number of chronic disabilities. Am J Med 1986;80:429-434.

8. Shibao C, Grijalva CG, Raj SR et al. Orthostatic hypotension-related hospitalizations in the United States. Am J Med 2007;120:975-980.

9. Eigenbrodt ML, Rose KM, Couper DJ et al. Orthostatic hypotension as a risk factor for stroke: The Atherosclerosis Risk in Communities (ARIC) study, 1987-1996. Stroke 2000;31:2307-2313.

10. Rose KM, Tyroler HA, Nardo CJ et al. Orthostatic hypotension and the incidence of coronary heart disease: The Atherosclerosis Risk in Communities Study. Am J Hypertens 2000;13(6 Pt 1):571-578.
11. Rose KM, Eigenbrodt ML, Biga RL et al. Orthostatic hypotension predicts mortality in middle-aged adults: The Atherosclerosis Risk in Communities (ARIC) Study. Circulation 2006;114:630-636.

12. Luukinen H, Koski K, Laippala P et al. Orthostatic hypotension and the risk of myocardial infarction in the home-dwelling elderly. J Intern Med 2004;255: 486-493.

13. Hossain M, Ooi WL, Lipsitz LA. Intra-individual postural blood pressure variability and stroke in elderly nursing home residents. J Clin Epidemiol 2001;54:488-494.

14. Luukinen H, Koski K, Laippala P et al. Prognosis of diastolic and systolic orthostatic hypotension in older persons. Arch Intern Med 1999;159: 273-280.

15. Weiss A, Beloosesky Y, Kornowski R et al. Influence of orthostatic hypotension on mortality among patients discharged from an acute geriatric ward. J Gen Intern Med 2006;21:602-606.

16. Luukinen $\mathrm{H}$, Airaksinen KE. Orthostatic hypotension predicts vascular death in older diabetic patients. Diabetes Res Clin Pract 2005;67: 163-166.

17. Masaki KH, Schatz IJ, Burchfiel CM et al. Orthostatic hypotension predicts mortality in elderly men: The Honolulu Heart Program. Circulation 1998; 98:2290-2295.

18. Davis BR, Langford HG, Blaufox MD et al. The association of postural changes in systolic blood pressure and mortality in persons with hypertension: The hypertension detection and follow-up program experience. Circulation 1987;75:340-346.

19. Hofman A, Breteler MM, van Duijn CM et al. The Rotterdam Study: Objectives and design update. Eur J Epidemiol 2007;22:819-829.

20. Bots ML, Looman SJ, Koudstaal PJ et al. Prevalence of stroke in the general population. The Rotterdam Study. Stroke 1996;27: 1499-1501.

21. van der Meer IM, Bots ML, Hofman A et al. Predictive value of noninvasive measures of atherosclerosis for incident myocardial infarction: The Rotterdam Study. Circulation 2004;109:1089-1094.

22. Bleumink GS, Knetsch AM, Sturkenboom MC et al. Quantifying the heart failure epidemic: Prevalence, incidence rate, lifetime risk and prognosis of heart failure. The Rotterdam Study. Eur Heart J 2004;25: 1614-1619.

23. Diabetes mellitus. Report of a WHO Study Group. World Health Organ Tech Rep Ser 1985;727:1-113.

24. WHO. International Statistical Classification of Diseases and Related Health Problems, 10 Ed. Geneva: WHO, 1992.

25. Remme WJ, Swedberg K. Guidelines for the diagnosis and treatment of chronic heart failure. Eur Heart J 2001;22:1527-1560.

26. Rutan GH, Hermanson B, Bild DE et al. Orthostatic hypotension in older adults. The Cardiovascular Health Study. CHS Collaborative Research Group. Hypertension 1992;19(6 Pt 1):508-519.

27. Smith JJ, Porth CM, Erickson M. Hemodynamic response to the upright posture. J Clin Pharmacol 1994;34:375-386.

28. Robertson D, DesJardin JA, Lichtenstein MJ. Distribution and observed associations of orthostatic blood pressure changes in elderly general medicine outpatients. Am J Med Sci 1998;315:287-295.

29. Kubo SH, Cody RJ. Circulatory autoregulation in chronic congestive heart failure: Responses to head-up tilt in 41 patients. Am J Cardiol 1983;52:512518.

30. Vinik AI, Maser RE, Mitchell BD et al. Diabetic autonomic neuropathy. Diabetes Care 2003;26:1553-1579. 